\title{
A Motor-Oriented Organization of Human Ventral Visual Cortex?
}

\author{
Jason P. Gallivan \\ Department of Psychology, Centre for Neuroscience Studies, Queen's University, Kingston, Ontario K7L 3N6, Canada \\ Review of Bracci and Peelen
}

The organizing principles of high-level primate occipitotemporal cortex (OTC) have puzzled cognitive neuroscientists for decades. Whereas visual response properties throughout the early visual system are highly structured and systematic, highlevel OTC appears to be arranged as a mosaic of specialized regions, each thought to be involved in the perceptual analysis of particular stimulus categories (e.g., objects, faces, scenes, bodies, etc.; Grill-Spector and Malach, 2004). Several theories have been proposed to account for this highly modular organization. According to one prominent view, the organization of OTC reflects a mapping based on similarities in the visual shape and form of objects (Haxby et al., 2001). Another view posits that this organization is a byproduct of weak visual field preferences throughout OTC (Levy et al., 2001). Notably, the majority of these theories, in some form or another, attempt to account for the organization of OTC on the basis of the visual structure of the world and/or how it is experienced. Given that many projections to OTC arise from early visual areas (Felleman and Van Essen, 1991), it is not surprising that most theories should attempt

Received Jan. 7, 2014; revised Jan. 15, 2014; accepted Jan. 16, 2014.

I am grateful for the support provided by the Banting Postdoctoral Fellowships program.

Correspondence should be addressed to Jason Gallivan, Department of Psychology, Centre for Neuroscience Studies, Botterell Hall, Queen's University, Kingston, Ontario K7L 3N6, Canada. E-mail: jasongallivan@gmail.com.

DOI:10.1523/JNEUROSCI.0060-14.2014

Copyright $\odot 2014$ the authors $\quad 0270-6474 / 14 / 343119-03 \$ 15.00 / 0$ to account for its organization on the basis of incoming visual information.

One compelling alternative to these accounts, however, argues that the organization of OTC emerges from the connectivity of OTC not just with early visual cortex but, more importantly, with the rest of the brain-particularly the "downstream" regions like parietal and somatomotor cortex that use OTC information to produce motor behavior (Mahon and Caramazza, 2011). In essence, this hypothesis argues that categorical specificity at the level of OTC may largely reflect a manifestation of the connectivity constraints imposed by a much more widely distributed network of areas (beyond that of the ventral visual pathway alone), with the different network specializations reflecting differences in how each stimulus category is ultimately processed, stored, and used by the entire brain to produce action. This hypothesis is provocative, but evidence in support of it is limited. Exciting recent findings in humans (Bracci and Peelen, 2013), however, appear consistent with this account and suggest that understanding the representation of object properties linked to action planning and control can provide insight into some of the organizational principles that governs functional specificity in OTC.

Bracci and Peelen (2013) provide evidence for an organization in lateral OTC (LOTC) based on how objects are controlled by the body. In particular, they show that representations of the body's effectors (e.g., hands) and objects used by the body as action effectors (e.g., hairbrush) are clustered together in LOTC. The authors performed four functional magnetic resonance imaging (fMRI) experiments in which they probed the visual responses of OTC to a wide range of effector and non-effector object stimuli. A clever feature of the experimental design was that the object stimuli used were independently rated, in separate behavioral experiments, on multiple action-related dimensions. These dimensions included: (1) body extension (i.e., the extent to which the object provides a physical extension of the body), (2) priming for hand movement (i.e., the extent to which the object naturally evokes hand/arm movement), (3) graspability (i.e., the extent to which the object is graspable with the hand), (4) hand-action centrality (i.e., the extent to which particular hand actions are characteristic of the object), and (5) hand association (the extent to which the object evokes imagery of the hands). Interestingly, Bracci and Peelen (2013) found that hand-selective regions in left LOTC showed heightened responses to objects used as action effectors that extend the body (e.g., tennis racquets). Additional analyses showed, among other things, that this same level of activity was not found for related stimuli (e.g., tennis balls) or other elongated stimuli not used for acting on other objects (e.g., guitars). Using multivoxel pattern analysis (MVPA), they further showed that the fMRI response 
patterns evoked by body-extending object stimuli were more correlated (and thus, more similar) to the patterns evoked by images of hands than those evoked by images of bodies. Finally, the authors demonstrated using whole-brain representational similarity analysis (in which fMRI response patterns associated with each exemplar item are correlated with that of every other exemplar item) that the activity patterns in left LOTC were more strongly predicted by how the objects were rated on the body extension dimension than the other object dimensions (hand priming, graspability, and handaction centrality).

These findings clearly suggest that the purported functionality of objects shape their representation in LOTC, but what is the basis for this body-object effector organization? One possibility is that the organization is grounded in well learned visual associations. For example, it has been suggested that the close clustering of face- and body-selective areas in ventral-temporal cortex might arise because these two visual stimuli are physically adjacent to one another in our typical experience of viewing others (i.e., faces are often seen atop bodies; Orlov et al., 2010). Similarly, hands and object-effectors (e.g., hairbrushes), are far more likely to statistically co-occur in the visual world than hands and other objects (e.g., clocks), and this might therefore explain why hands and object-effectors are clustered together in OTC. Such clustering is found throughout the early visual system and is thought to be an organizational principle that minimizes wiring length and promotes efficient communication between frequently coactivated neurons (Graziano and Aflalo, 2007). This general organizational principle, however, appears to also extend to the motor system. Many of the features of movement that statistically cooccur in our naturalistic behavioral repertoire (e.g., stereotyped movements for eating) appear to be clustered together in coarse topographic maps that span the supplementary, motor, and premotor cortices (Graziano and Aflalo, 2007). Given the ecologically rooted notion that OTC did not originally evolve for the purposes of pure perceptual processing but rather for a more fundamental role in extracting information from the environment for the purposes of action and survival (Cisek, 2007), it seems plausible that OTC, in part, might be organized with respect to implementing motor behavior.

In light of this consideration, a second possibility is that the results of Bracci and Peelen (2013) reflect features of a much more motor-oriented organization in
OTC whereby effectors used for generating similar types of actions are clustered together. In this sense, rather than the LOTC organization reflecting our visual experience as passive observers of the world (as with the first possibility), it instead reflects how we use items to perform behaviors as actors in the world. With respect to this second, more provocative possibility, previous work reporting the encoding of hand, tool, and other bodyrelated movements in LOTC seems particularly relevant.

Previous fMRI studies have reported activity in body-related representations in LOTC during the execution of movements, with some of this work indicating a shared topographic representation in OTC between moved and passively viewed body parts (Orlov et al., 2010). In addition, more recent work using MVPA has suggested a role for LOTC in movement planning, showing that upcoming object-directed movements of the hand and those performed with a tool can be decoded, respectively, from pre-movement fMRI activity patterns in a body-selective region, the extrastriate body area, and a tool-selective region, the posterior middle temporal gyrus (pMTG; Gallivan et al., 2013). Notably, this pMTG region, defined by its preferred responses to passively viewed tool stimuli (Gallivan et al., 2013), is located in a near-identical location to the LOTC region of Bracci and Peelen (2013).

Further evidence suggesting a motororiented organization in LOTC comes from comparing the foci of activity in Bracci and Peelen (2013) to that of other well documented OTC regions. Critically, whereas the stimulus categories of objects, scenes, faces, and bodies appear to be more robustly represented in right than left OTC (GrillSpector and Malach, 2004), the LOTC area of Bracci and Peelen (2013) is localized to the left hemisphere only. Neuropsychological and neurophysiological investigations suggest that the left hemisphere is specialized for visual-motor control (Serrien et al., 2006) and, given that all the participants tested in the Bracci and Peelen (2013) study were right-hand dominant, these individuals would be expected to have particularly well developed visual-motor networks in the left (contralateral) hemisphere. The above evidence, when taken together, suggests that left LOTC may actually play some role in action planning and control. But what is this role?

According to the highly influential dual visual streams framework (Goodale and Milner, 1992), it is frontoparietal cortex, not
OTC, that is involved in extracting the action-relevant features of objects for the purposes of motor behavior. Nevertheless, to produce the appropriate set of motor commands upon an object, we must first recognize that object and then access stored knowledge concerning its use-a process likely to require the retrieval of more stable, long-term object representations contained in OTC (Goodale and Milner, 1992). This OTC-frontoparietal cross talk could be supported by interconnections between OTC areas involved in object recognition and parietal areas involved in grasping (Borra et al., 2008). This, however, would only suggest an indirect role for OTC in motor behavior. Nevertheless, this same OTC-parietal interconnectivity would also allow OTC, in principle, to gain access to efference copies of motor commands (from parietal cortex). Due to the delay of incoming sensory information, efference signals allow neural systems to predict (and cancel out) the visual and proprioceptive consequences of selfgenerated movement and they feature prominently in models of motor control (Wolpert and Flanagan, 2001). Thus, one possibility is that the LOTC area of Bracci and Peelen (2013) may have originally evolved to participate in motor behavior by receiving, during tool-related actions, efference signals (from parietal cortex) related to planned hand movements. This would allow LOTC to disambiguate sensory signals related to self-generated hand movements versus movements of the tool in the hand (e.g., slippage) and, in this way, possibly play a key role- through its cross talk with parietal cortex-in implementing corrective actions (e.g., increasing the forces applied to the tool).

In addition to the visual- versus motor-related nature of the LOTC organization noted above, several other important questions stem from the study of Bracci and Peelen (2013). What are the evolutionary origins of this LOTC region? Studies in nonhuman primates suggest that homologous OTC regions may exist for face-, scene-, and body-processing, but what about objects used as effectors (e.g., tools)? Also, critical to the discussion above, how does the object-effector LOTC region interact and share information with areas positioned closer to the final motor pathways, like parietal and premotor cortex? What are the response properties of LOTC during tasks requiring real actions upon objects? Indeed, while much of the field focuses on characterizing and partitioning the activity of OTC during passive viewing tasks [as in Bracci and Peelen (2013)], it will be criti- 
cal for future work to explore the functions of OTC in the generation of real object-oriented behaviors, like grasping and manipulation.

In summary, the results of Bracci and Peelen (2013) offer key insights into what may be some of the organizing principles of OTC. These findings highlight the need for understanding (and interpreting) OTC activity within the greater context of the whole-brain networks in which they are embedded and the types of neural computations they are likely to support. From this standpoint, future work is needed to explore the connectivity of OTC regions both with each other and with the rest of the brain, and investigate how OTC supports the planning and control of everyday behaviors.

\section{References}

Borra E, Belmalih A, Calzavara R, Gerbella M, Murata A, Rozzi S, Luppino G (2008) Cor- tical connections of the macaque anterior intraparietal (AIP) area. Cereb Cortex 18: 1094-1111. CrossRef Medline

Bracci S, Peelen MV (2013) Body and object effectors: the organization of object representations in high-level visual cortex reflects body-object interactions. J Neurosci 33: 18247-18258. CrossRef Medline

Cisek P (2007) Cortical mechanisms of action selection: the affordance competition hypothesis. Phil Trans R Soc Lond B Biol Sci 362: 1585-1599. CrossRef Medline

Felleman DJ, Van Essen DC (1991) Distributed heirarchical processing in the primate cerebral cortex. Cereb Cortex 1:1-47. CrossRef Medline

Gallivan JP, McLean DA, Valyear KF, Culham JC (2013) Decoding the neural mechanisms of human tool use. eLife 2:e00425. CrossRef Medline

Goodale MA, Milner AD (1992) Separate visual pathways for perception and action. Trends Neurosci 15:20-25. CrossRef Medline

Graziano MS, Aflalo TN (2007) Mapping behavioral repertoire onto the cortex. Neuron 56:239-251. CrossRef Medline

Grill-Spector K, Malach R (2004) The human visual cortex. Annu Rev Neurosci 27:649677. CrossRef Medline

Haxby JV, Gobbini MI, Furey ML, Ishai A, Schouten JL, Pietrini P (2001) Distributed and overlapping representations of faces and objects in ventral temporal cortex. Science 293:2425-2430. CrossRef Medline

Levy I, Hasson U, Avidan G, Hendler T, Malach R (2001) Center-periphery organization of human object areas. Nat Neurosci 4:533-539. Medline

Mahon BZ, Caramazza A (2011) What drives the organization of object knowledge in the brain? Trends Cogn Sci 15:97-103. CrossRef Medline

Orlov T, Makin TR, Zohary E (2010) Topographic representation of the human body in the occipitotemporal cortex. Neuron 68:586600. CrossRef Medline

Serrien DJ, Ivry RB, Swinnen SP (2006) Dynamics of hemispheric specialization and integration in the context of motor control. Nat Rev Neurosci 7:160-166. CrossRef Medline

Wolpert DM, Flanagan JR (2001) Motor prediction. Curr Biol 11:R729-R732. CrossRef Medline 